\title{
Genome wide study of tardive dyskinesia in schizophrenia
}

\author{
Keane Lim (1)', Max Lam (1) ${ }^{1,2,3}$, Clement Zai ${ }^{4}$, Jenny Tay ${ }^{1}$, Nina Karlsson”, Smita N. Deshpande ${ }^{6}$, B. K. Thelma7, \\ Norio Ozaki $\mathbb{1}^{8}$, Toshiya Inada $\mathbb{1}^{9}$, Kang Sim $\mathbb{1}^{1,10}$, Siow-Ann Chong ${ }^{1,11}$, Todd Lencz $\mathbb{B}^{2,3,12}$, Jianjun Liu $\mathbb{C}^{5,13}$ and \\ Jimmy Lee (i) ${ }^{1,11,14}$
}

\begin{abstract}
Tardive dyskinesia (TD) is a severe condition characterized by repetitive involuntary movement of orofacial regions and extremities. Patients treated with antipsychotics typically present with TD symptomatology. Here, we conducted the largest GWAS of TD to date, by meta-analyzing samples of East-Asian, European, and African American ancestry, followed by analyses of biological pathways and polygenic risk with related phenotypes. We identified a novel locus and three suggestive loci, implicating immune-related pathways. Through integrating trans-ethnic fine mapping, we identified putative credible causal variants for three of the loci. Post-hoc analysis revealed that SNPs harbored in TNFRSF1B and CALCOCO1 independently conferred three-fold increase in TD risk, beyond clinical risk factors like Age of onset and Duration of illness to schizophrenia. Further work is necessary to replicate loci that are reported in the study and evaluate the polygenic architecture underlying TD.
\end{abstract}

\section{Introduction}

Tardive Dyskinesia (TD) is a persistent and potentially debilitating involuntary movement disorder characterized by choreiform, athetoid, and or dystonic movements ${ }^{1,2}$. TD is largely caused by antipsychotic treatment and was first described in $1957^{3}$. Although commonly observed in patients with schizophrenia, TD can occur in individuals with other psychiatric disorders, as long as they have been similarly exposed to prolonged antipsychotic treatment. The prevalence of TD in schizophrenia is estimated to be between 15 and 30\%, although rates of TD have reduced with prescription of second-generation antipsychotics ${ }^{4-6}$. Nevertheless, second-generation antipsychotics still carry with them a risk of developing TD, and TD remains a clinically relevant phenotype as it has been associated with

\footnotetext{
Correspondence: Jianjun Liu (liuj3@gis.a-star.edu.sg) or

Jimmy Lee (Jimmy_lee@imh.com.sg)

${ }^{1}$ Research Division, Institute of Mental Health, Singapore, Singapore

${ }^{2}$ Zucker Hillside Hospital, New York, NY, USA

Full list of author information is available at the end of the article

These authors contributed equally: Keane Lim, Max Lam

These authors jointly supervised this work: Jianjun Liu, Jimmy Lee
}

more severe schizophrenia illness, cognitive impairments, lowered quality of life and increased mortality ${ }^{7-10}$.

The pathophysiology of TD is currently unknown. Theories pertaining to dopamine receptor hypersensitivity, serotonergic dysfunction, GABA insufficiency, and free radical damage have been put forth ${ }^{2,11,12}$. It is postulated that TD is related to the schizophrenia disease process; recent reports have linked basal ganglia (caudate nucleus) volume reductions to TD in schizophrenia; notably, these samples were on second-generation antipsychotic-suggesting the pathological process towards TD is beyond neurochemical properties ${ }^{13}$. As TD is potentially irreversible with no effective treatment, there needs to be an added emphasis on the prevention and identification of genetic risk factors. Several genes (e.g.-, $D R D 2, D R D 3$, $M n S O D, C Y P 2 D 6$, GRIN2A, and GRIN2B) have been implicated in candidate gene studies, but replication remains equivocal ${ }^{14-18}$. Genome-Wide Association Studies (GWAS) performed on the TD phenotype suggested that the GLI family zinc finger 2 (GLI2), heparan sulfate proteoglycan 2 (HSPG2), dipeptidyl-peptidase 6 (DPP6), and GABA pathway genes could putatively be considered

\section{(c) The Author(s) 2021}

\footnotetext{
(c) (i) Open Access This article is licensed under a Creative Commons Attribution 4.0 International License, which permits use, sharing, adaptation, distribution and reproduction in any medium or format, as long as you give appropriate credit to the original author(s) and the source, provide a link to the Creative Commons license, and indicate if changes were made. The images or other third party material in this article are included in the article's Creative Commons license, unless indicated otherwise in a credit line to the material. If material is not included in the article's Creative Commons license and your intended use is not permitted by statutory regulation or exceeds the permitted use, you will need to obtain permission directly from the copyright holder. To view a copy of this license, visit http://creativecommons.org/licenses/by/4.0/.
} 
susceptibility genes for $\mathrm{TD}^{19-23}$. Nonetheless, these studies are limited by small samples and require further replication. Here, we report the findings of the largest GWAS of TD to date. We identified a novel locus at 16q24.1 $\left(P=3.01 \times 10^{-8}\right)$ and three suggestive loci $(1 \mathrm{p} 36.22$, 6q23.2, 12q13.13; $P<5 \times 10^{-7}$ ) associated with TD.

\section{Methodology}

Participants from the Singapore Clinical and Translational Research program in Singapore, and the Clinical Antipsychotic Trials of Intervention Effectiveness $(\text { CATIE })^{24-26}$ study were included in the current report. All participants met criteria for DSM-IV diagnosis for schizophrenia. Tardive dyskinesia was ascertained via the Abnormal Involuntary Movement Scale (AIMS) ${ }^{27,28}$. After quality control procedures, 1406 participants (280 with TD), and 6,291,020 SNPs remained. Linear Mixed Models GWA was performed via GEMMA ${ }^{29}$, and independent cohorts across the two studies were meta-analyzed via fixed effects inverse variance approach in METAL ${ }^{30}$. GWAS summary statistics were then subjected to functional annotation for GWAS identified $\operatorname{loci}^{31}$, eQTL lookups, pathway analysis ${ }^{32}$, and transcriptome-wide analysis ${ }^{33}$, which were conducted to characterize potential biological mechanisms underlying TD. Further finemapping analysis was also carried out to identify credible causal variants ${ }^{34-36}$. A series of polygenic risk score analyses were conducted to compare the genetic architecture of TD with related phenotypes ${ }^{37}$. We also carried out a series of post-hoc multivariate logistic regression analysis to investigate joint clinical and genetic factors that predict the emergence of TD. Finally, variants previously associated with TD were compared with the current GWAS results ${ }^{11,18,22,23,38-46}$. Detailed methodological approaches are further reported in the Supplementary Information included with the current report.

\section{Results}

\section{Demographics and assessment of tardive dyskinesia}

Demographics are reported in Table 1 and Supplementary Table 1 . There were $71.1 \%$ males and $28.9 \%$ females with TD. There was no significant difference in gender proportion between individuals with TD and those without, $X^{2}(1, n=1406)=0.142, P=0.706$. There were significant differences in age, $t(1404)=-14.06, P=4.0 \times 10^{-42}$, age of illness onset, $t(392.7)=-3.06, P=0.0024$, duration of illness, $t(1390)=-11.28, P=2.76 \times 10^{-28}$, and antipsychotic dose measured in CPZ equivalent, $t(516.3)=3.73, P=$ 0.002 between individuals with and without TD. These differences in demographics and clinical characteristics were further modelled using a polygenic risk score approach that allows us to examine genetic effects for TD alongside demographics effects; results are reported in subsequent sections.
Table 1 Characteristics of tardive dyskinesia versus nontardive dyskinesia samples.

\begin{tabular}{llc}
\hline & $\begin{array}{l}\text { TD samples } \\
(\boldsymbol{N}=\mathbf{2 8 0})\end{array}$ & $\begin{array}{l}\text { Non-TD samples } \\
(\boldsymbol{N}=\mathbf{1 1 2 6})\end{array}$ \\
\hline Gender (male/female) & $199 / 81$ & $813 / 313$ \\
Age (years) & $55.51(11.38)$ & $44.23(12.17)$ \\
Age of illness onset & $28.71(10.71)$ & $26.55(9.50)$ \\
(years) & & $17.22(12.23)$ \\
Duration of Illness (years) & $26.52(12.55)$ & $13.51(5.99)$ \\
PANSS (score) & & $15.69(6.99)$ \\
Positive symptoms & $12.90(6.01)$ & $27.96(10.12)$ \\
Negative symptoms & $15.42(6.89)$ & \\
General & $27.44(10.11)$ & $57.17(20.40)$ \\
psychopathology & & \\
Total & $55.76(20.67)$ & $487(43.3 \%)$ \\
Antipsychotics, $n$ (\%) & & $394(35.0 \%)$ \\
Typical only & $170(60.7 \%)$ & $93(8.3 \%)$ \\
Atypical only & $66(23.6 \%)$ & $7(0.6 \%)$ \\
Typical + atypical & $17(6.1 \%)$ & $637.16(679.07)$ \\
None & $2(0.7 \%)$ & \\
$\begin{array}{l}\text { Daily CPZ equivalent } \\
\text { dose, mg (SD) }\end{array}$ & $494.7(494.30)$ & \\
Total aims score & $11.09(5.11)$ & \\
\hline
\end{tabular}

\section{Genome-wide association of tardive dyskinesia}

Standard GWAS quality control procedures were carried out (Supplementary Figs. 1-5). We carried out Principal Components Analysis (PCA) on each ancestry group to identify overall population stratification across samples, and, within population PCA to identify fine-grain population outliers (See Supplementary Information). We removed population outliers detected by PCA via a series of $k$-means clustering. Notably, association analysis was carried out within each ancestry first and thereafter metaanalyzed. Linear mixed models conducted via the GEMMA $^{29}$ package revealed significant genome-wide association of TD at the level of the CATIE cohorts, but not the STCRP cohort (Supplementary Fig. 6). Subsequent fixed-effect inverse variance meta-analysis ${ }^{30}$ $\left(\lambda_{\mathrm{GC}}=1.02\right.$; Table 2, Figs. 1-3) across the STCRP and CATIE cohorts revealed one novel locus on chromosome 16 (rs11639774, downstream of GSE1) $\left(P=3.01 \times 10^{-8}\right)$. Three other suggestive independent genomic loci $(P<5 \times$ $10^{-7}$ ) were identified on chromosome 1 (rs499646, $P=$ $8.30 \times 10^{-8}$, TNFRSF1B), chromosome 6 (rs6926250, $P=$ $\left.2.54 \times 10^{-7}, E P B 41 L 2\right)$, and chromosome 12 (rs4237808, $\left.P=1.08 \times 10^{-7}, C A L C O C O 1\right)$. Due to low minor allele frequencies in the STCRP sample, two of the SNPs were 
Table 2 Top SNPs associated with tardive dyskinesia.

\begin{tabular}{|c|c|c|c|c|c|c|c|c|c|c|c|}
\hline SNP & Symbol & Location & Position & Variant & Effect allele & Other allele & EAF & Beta & SE & $P$-value & HetP \\
\hline rs11639774 & GSE1 & $16 q 24.1$ & 85221868 & Intergenic & A & G & 0.168 & 0.151 & 0.0273 & 3.01E-08 & 0.067 \\
\hline rs499646 & TNFRSF1B & $1 p 36.22$ & 12239089 & Intronic & A & G & 0.12 & 0.1949 & 0.0364 & 8.30E-08 & 0.246 \\
\hline rs4237808 & CALCOCO1 & $12 q 13.13$ & 54093797 & Intergenic & $\mathrm{T}$ & C & 0.4914 & 0.0765 & 0.0144 & $1.08 \mathrm{E}-07$ & 0.506 \\
\hline rs6926250 & EPB41L2 & $6 \mathrm{q} 23.2$ & 131302473 & Intronic & $\mathrm{T}$ & C & 0.6872 & -0.0968 & 0.0188 & 2.54E-07 & 0.447 \\
\hline
\end{tabular}

SNP single-nucleotide polymorphism, EAF effect allele frequency, $S E$ standard error, HetP heterogenity $P$-value.

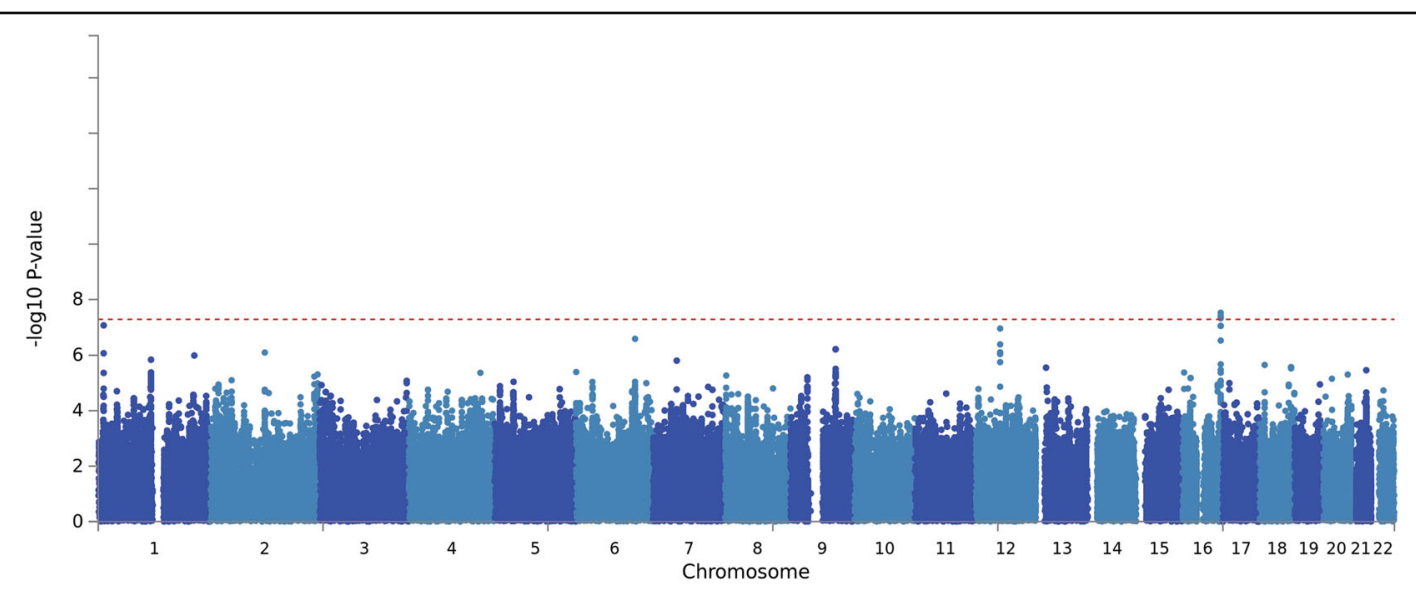

Fig. 1 Manhattan plot for GWAS fixed effect meta-analysis of TD. Note: Three cohorts were included in the fixed-effect meta-analysis, STCRP, CATIE-EUR, and CATIE-AFR. A single GWAS signal was found at 16q24.1 for rs11639774.

only present in the CATIE cohorts. Proxy SNPs in LD within the STCRP cohort were identified using the SNP Annotation and Proxy Search (SNAP) ${ }^{47}$. Further metaanalysis of association results from both proxy SNPs were carried out using Fisher's $p$-value meta-analysis approach (https://CRAN.R-project.org/package=metap). Fisher's meta-analysis for the top SNP (rs11639774) and proxy SNP (rs9928615) in the STCRP cohort was significant $\left(X^{2}(4)=40.28, P=3.79 \times 10^{-08}\right)$. Similarly, results for SNP (rs499626) and proxy SNP (rs11569835) in the STCRP cohort reached suggestive significance $\left(\chi^{2}(4)=\right.$ $36.48, P=2.31 \times 10^{-07}$ ), with both primary variants with proxy SNP meta-analysis showing consistent effects (Supplementary Table 2). GWAS markers were further annotated via ANNOVAR, eQTL, Chromatin Interaction modules within the Functional Mapping and Annotation of Genome-Wide Association Studies (FUMA) ${ }^{31}$ (Supplementary Tables 3, 4; Supplementary Fig 7).

\section{Gene-based and pathway analysis}

MAGMA was used to conduct both the gene-based and geneset analysis ${ }^{32}$. Gene-based analysis tests for association

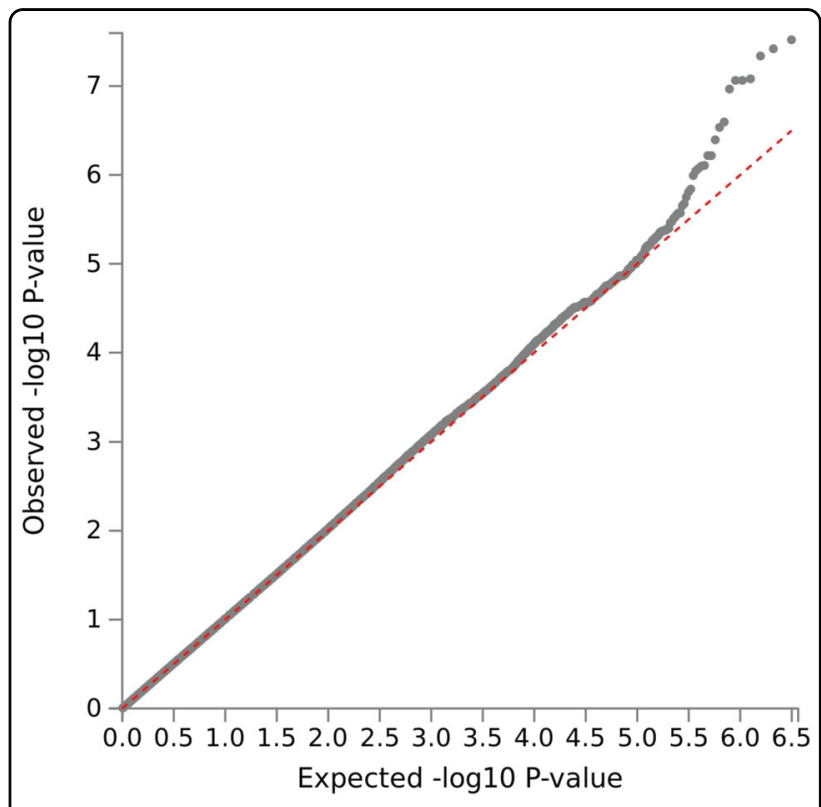

Fig. 2 QQ plot for meta-analysis of STCRP, CATIE-EUR, and CATIEAFR. Note: Lambda $=1.02$ 
(a)
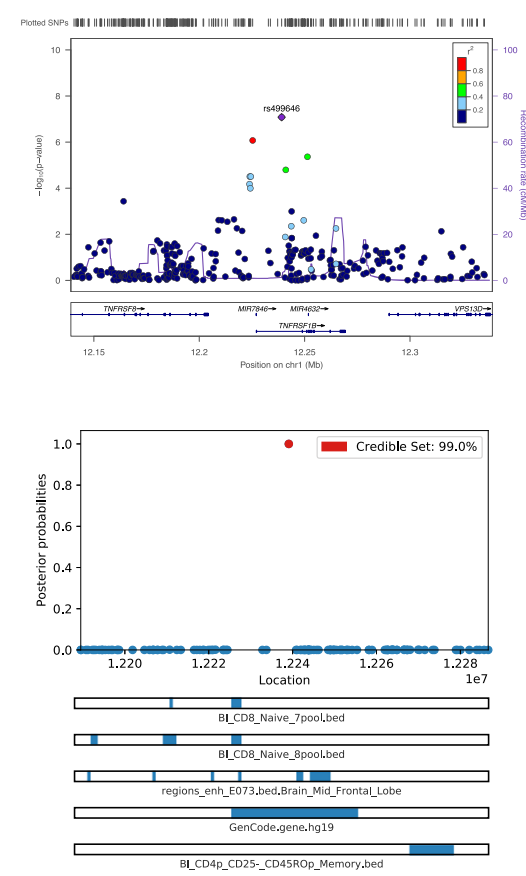

(b)
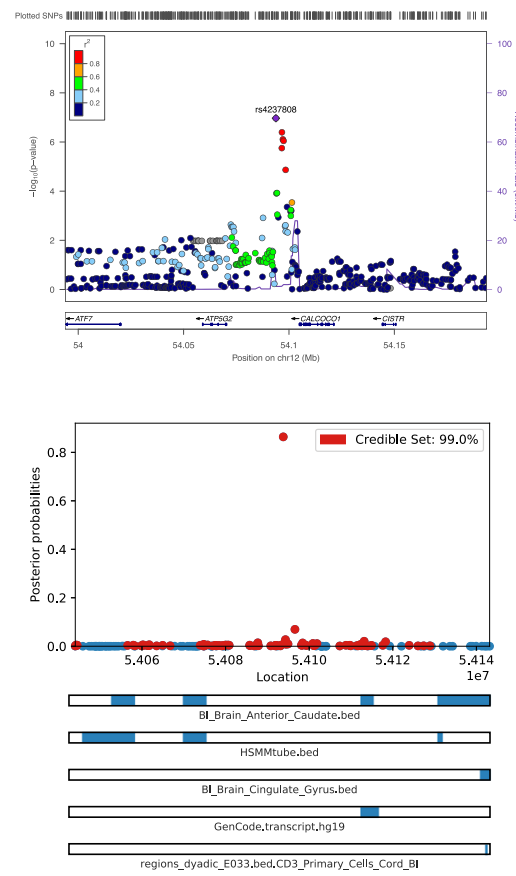

(c)
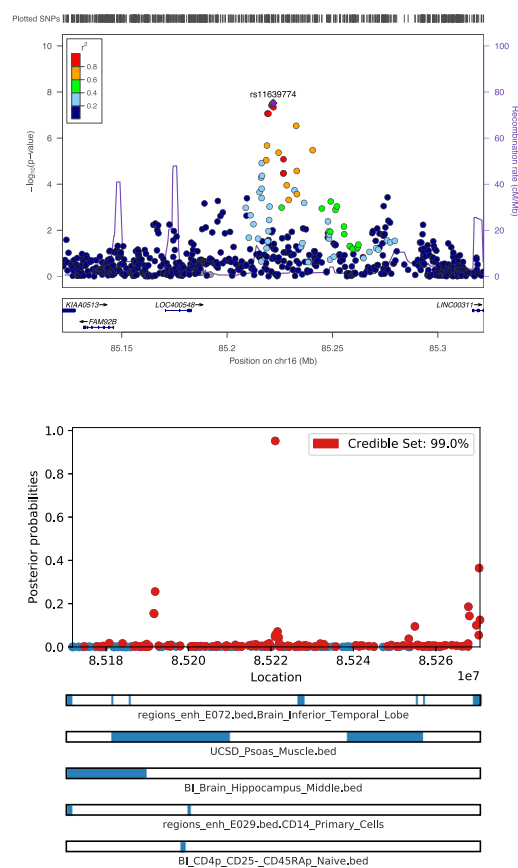

Fig. 3 Regional loci and fine-mapping plots. Top panel represents regional plots for a Chromosome 1, b Chromosome 12, and c Chromosome 16. Bottom panel represents the visualization of $99 \%$ credible SNP set for a Chromosome 1, b Chromosome 12, and c Chromosome 16, against location of SNP, with top annotation bars.

of TD with markers within the gene by mapping the SNPs to gene level, while geneset analysis aggregates individual genes to a collection of genes with overlapping characteristics $^{28}$. Results of MAGMA gene-based analysis on 18,259 mapped autosomal genes after Bonferroni correction are presented in Supplementary Table 5. None of the genes were significant after Bonferroni correction. MAGMA competitive geneset analysis ${ }^{32}$ was conducted using the most recent Molecular Signature Database version $6.1^{48}$. Although none of the 17,199 genesets survived Bonferroni correction, top pathways implicated regulation of immunoglobulin production and immunoglobulin isotype switching, expression of chemokine receptors, and regulation of cell growth, and monocytes (Supplementary Table 6).

\section{eQTL lookups/transcriptome-wide analysis}

Expression quantitative trait loci (eQTL) were performed as part of FUMA ${ }^{31}$ GWAS pipeline. Notably, eQTL effect of ATP5G2 (Bonferroni corrected $P=1.24 \times$ $10^{-14}$, Supplementary Table 7) and MAP3K12 (Bonferroni corrected $p=6.29 \times 10^{-10}$, Supplementary Table 7) was observed for subthreshold genomic significance loci for 12q13.13, TNFRS1B (Bonferroni corrected $p=4.60 \times$ $10^{-08}$, Supplementary Table 7) for $1 \mathrm{p} 36.22$, EPB41L2 (Bonferroni corrected $p=2.86 \times 10^{-07}$, Supplementary
Table 7) and AKAP7 (Bonferroni corrected $p=1.48 \times$ $10^{-06}$, Supplementary Table 7) for 6q23.2, with expression in various tissues, including those related to motor functions (Table 3).

Transcriptome-wide analysis was implemented via MetaXcan ${ }^{33}$. Unlike eQTL lookups, the MetaXcan approach further incorporates evidence from GWAS summary statistics with genome-wide gene expression profiles from the GTex ${ }^{49}$ database. This provides information of potential functional enrichment within a genomic locus. Here, we found lower expression of a transcript, $E P B 41 L 2$, in the esophagus muscularis at Bonferronicorrected significance $(P=0.02$, Supplementary Table 8$)$.

\section{Fine-mapping analysis}

Cross-Ancestry fine mapping was performed on PAINTOR v3.1 $1^{34,35}$ to identify putative causal variants on the 4 loci (1p36.22, 6q23.2, 12q13.13, and 16q24.1). The 99\% cumulative posterior probability identified a total of 231 putative credible causal SNPs across three loci (1p36.22, 12q13.13, and 16q24.1; Supplementary Table 9, Supplementary Fig. 8). From these, highly credible SNPs were defined as posterior probability $>0.8$. This identified a putative causal variant for each locus: 1 p36.22 (rs499646, posterior probability $=0.99), \quad 12 \mathrm{q} 13.13$ $(\mathrm{rs} 4237808$, posterior probability $=0.863)$, and $16 \mathrm{q} 24.1$ 
Table 3 Summary of expression quantitative trait loci (eQTL) analysis.

\begin{tabular}{|c|c|c|c|}
\hline Symbol & Chr & Tissue & Database \\
\hline TNFRSF1B & 1 & BIOS_eQTL_geneLevel & BIOSQTL \\
\hline AKAP7 & 6 & BIOS_eQTL_geneLevel & BIOSQTL \\
\hline AKAP7 & 6 & Muscle_Skeletal & GTEx_v7 \\
\hline AKAP7 & 6 & Thyroid & GTEx_v7 \\
\hline EPB41L2 & 6 & xQTLServer_eQTLs & xQTLServer \\
\hline EPB41L2 & 6 & Skin_Sun_Exposed_Lower_leg & GTEx_v7 \\
\hline EPB41L2 & 6 & Esophagus_Muscularis & GTEx_v7 \\
\hline EPB41L2 & 6 & Esophagus_Mucosa & GTEx_v7 \\
\hline EPB41L2 & 6 & Lung & GTEx_v7 \\
\hline ATF7 & 12 & BIOS_eQTL_geneLevel & BIOSQTL \\
\hline ATP5G2 & 12 & Esophagus_Mucosa & GTEx_v7 \\
\hline ATP5G2 & 12 & BIOS_eQTL_geneLevel & BIOSQTL \\
\hline ATP5G2 & 12 & Thyroid & GTEx_v7 \\
\hline ATP5G2 & 12 & Nerve_Tibial & GTEx_v7 \\
\hline ATP5G2 & 12 & Adipose_Subcutaneous & GTEx_v7 \\
\hline ATP5G2 & 12 & Brain_Cerebellar_Hemisphere & GTEx_v7 \\
\hline ATP5G2 & 12 & Brain_Cerebellum & GTEx_v7 \\
\hline ATP5G2 & 12 & Testis & GTEx_v7 \\
\hline ATP5G2 & 12 & Esophagus_Muscularis & GTEx_v7 \\
\hline ATP5G2 & 12 & Adipose_Visceral_Omentum & GTEx_v7 \\
\hline ATP5G2 & 12 & Small_Intestine_Terminal_Ileum & GTEx_v7 \\
\hline ATP5G2 & 12 & Lung & GTEx_v7 \\
\hline ATP5G2 & 12 & Skin_Not_Sun_Exposed_Suprapubic & GTEx_v7 \\
\hline ATP5G2 & 12 & Artery_Tibial & GTEx_v7 \\
\hline ATP5G2 & 12 & CRBL & BRAINEAC \\
\hline ATP5G2 & 12 & MEDU & BRAINEAC \\
\hline ATP5G2 & 12 & PUTM & BRAINEAC \\
\hline ATP5G2 & 12 & SNIG & BRAINEAC \\
\hline ATP5G2 & 12 & FCTX & BRAINEAC \\
\hline ATP5G2 & 12 & aveALL & BRAINEAC \\
\hline ATP5G2 & 12 & TCTX & BRAINEAC \\
\hline ATP5G2 & 12 & WHMT & BRAINEAC \\
\hline ATP5G2 & 12 & OCTX & BRAINEAC \\
\hline ATP5G2 & 12 & THAL & BRAINEAC \\
\hline ATP5G2 & 12 & HIPP & BRAINEAC \\
\hline ESPL1 & 12 & Esophagus_Mucosa & GTEx_v7 \\
\hline MAP3K12 & 12 & BIOS_eQTL_geneLevel & BIOSQTL \\
\hline NPFF & 12 & BIOS_eQTL_geneLevel & BIOSQTL \\
\hline SP1 & 12 & Esophagus_Mucosa & GTEx_v7 \\
\hline SP7 & 12 & Thyroid & GTEx_v7 \\
\hline SP7 & 12 & Testis & GTEx_v7 \\
\hline
\end{tabular}

$($ rs28468398, posterior probability $=0.952)$. Notably, for loci on 1p36.22 and 12q13.13, the index SNP identified in GWAS is also a putative causal SNP identified by PAINTOR (See Fig. 3). Further annotations via the Variant Effect Predictor ${ }^{50}$ revealed that (i) rs499646 is an intronic variant $3500 \mathrm{bp}$ from the promotor of TNFRSF1B and is a known protein-coding variant and appears to be a loss-of-function intolerant variant, (ii) rs4237808 is an intergenic variant between ATP5G2 and CALCOCO1, but lies within $1000 \mathrm{bp}$ of two CTCF binding sites, and $3200 \mathrm{bp}$ from a known promotor flank, and (iii) rs28468398 is a regulatory region variant, which lies within a transcription factor binding site on CTC786C10.1/GSE1 gene. Notably, additional joint finemap annotations revealed that all three variants were enriched by known brain level gene expression sites close by (See Fig. 3). GCTA-COJO ${ }^{36}$ revealed no further signals present in the loci beyond independent variants identified via $L D$ clumping or fine mapping (Supplementary Fig. 9).

\section{Polygenic risk modelling of TD with other diseases and traits}

Polygenic risk score modelling via PRSice2 ${ }^{37}$ (Supplementary Fig. 10) revealed best polygenic association of TD with amyotrophic lateral Sclerosis (ALS), albeit only two PRS thresholds remained significant after Bonferroni correction $\left(\mathrm{P}_{\mathrm{T}}=0.5 ; \mathrm{P}_{\mathrm{T}}=1.0\right)$. ALS PRS-based pathway analysis indicated "misfolded protein" as a top pathway shared between TD and ALS (Supplementary Fig. 11), although this remains a trend finding. We followed up on the possibility that there might be pleiotropic genetic effects of ALS and TD, given that both conditions implicated motor neurons. A post-hoc lookup of ALS variants and genes was carried out on GWAS catalog. We filtered GWAS SNPs previously reported to be associated with ALS based on $p<1 \mathrm{e}-6$ and extracted corresponding mapped genes. These were then looked up in the MAGMA gene results reported earlier. We found that three previously known ALS genes, FBXO15, FAM19A1, and NP5 genes were nominally associated with TD genes (TD Gene P-values: FBXO15: 0.0210, FAM19A1: 0.0356 and NPS: 0.0455). Cross-Trait polygenic risk scores were also conducted on other psychopathological or autoimmune traits-such as schizophrenia, bipolar disorder, Alzheimer's disease, Parkinson's disease, depression, rheumatoid arthritis, and Crohn's disease but were not significant after multiple testing correction. It is, however, notable that aspect of the schizophrenia and rheumatoid arthritis did appear to be close to the multiple correction threshold for $P_{\mathrm{T}}<0.05$.

\section{Post-hoc clinical analysis: effects of nedication type and SNP effects on TD}

We performed post-hoc multivariate logistic regression analysis to investigate potential effects of clinical factors affecting TD beyond genetic effects. Preliminary linear-bylinear Chi-square analysis were significant $\left(X^{2}=18.67, p=\right.$ $1.40 \times 10^{-5}$, see Fig. 4a). Expectedly, there was a significant proportion of TD cases taking typical, compared to atypical antipsychotics. The Clinical Baseline model (Age of Onset, Duration of illness, Daily CPZ equivalents) was significantly associated with TD, for individuals who were taking either typical $\left(\chi^{2}=125.71, \mathrm{df}=25, p=2.15 \times 10^{-15}\right)$ or atypical antipsychotics $\left(\chi^{2}=75.57, \mathrm{df}=25, p=5.55 \times 10^{-7}\right)$. There were no significant model differences between the Clinical 
(a)

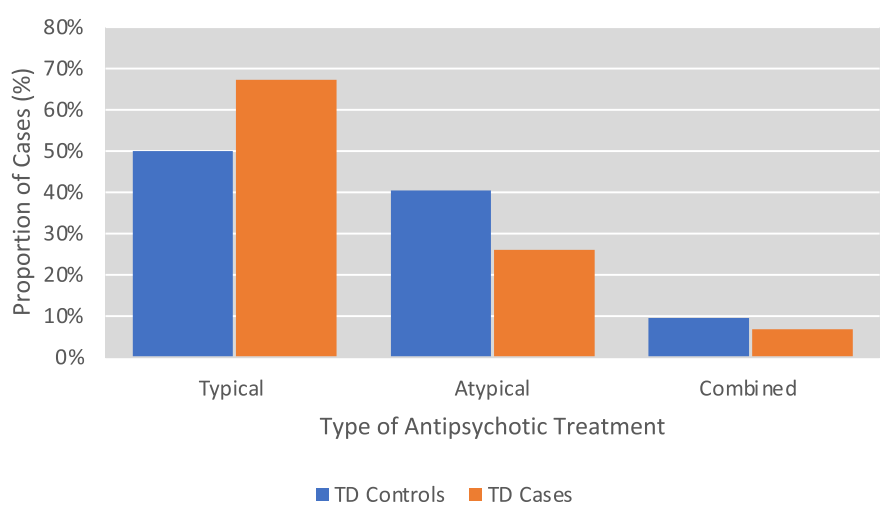

(b)

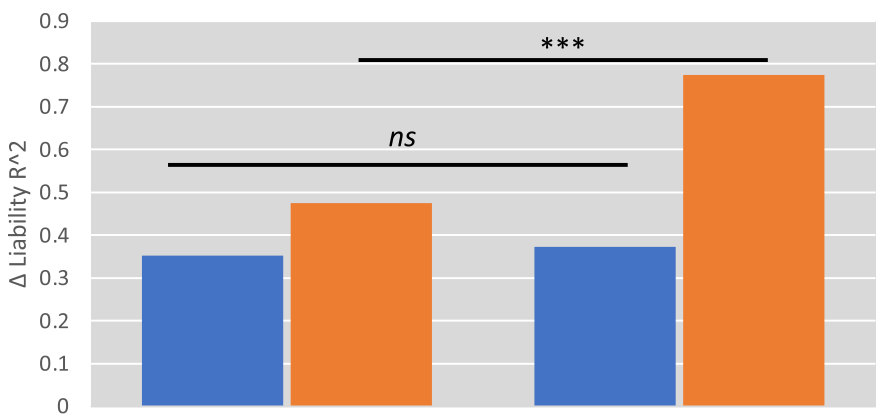

$\Delta$ Clinical Baseline

$\Delta$ Clinical + Genetic

- Typical Antipsychotics

Atypical Antipsychotics

(c)

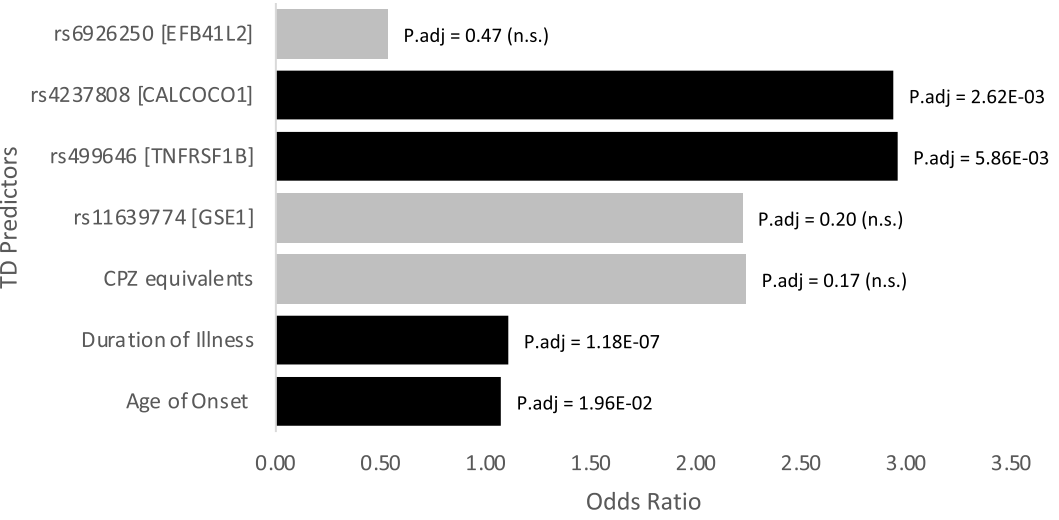

Fig. 4 Clinical and genetic risk factors for TD. a Stratified analysis for antipsychotic type and TD $\mathbf{b}$ Logistic regression model for Clinical Baseline versus Clinical + Genetic Model. Y-axis: Liability scale R² for logistic regression model, case-control proportions adjusted for TD prevalence set at $15 \%$. Clinical Baseline: Age of Onset, Duration of Illness, Daily CPZ equivalents. Clinical + Genetic: includes Clinical Baseline + rs6926250, rs4237808, rs499646, rs 11639774. ${ }^{* * *} p=7.09$ e-8. c Odds ratio for predictors in Clinical + Genetic model. Black colored bars are significant after Bonferroni correction for all variables entered in the logistic regression model.

Baseline model nor Clinical + Genetic model (Clinical Baseline + rs6926250, rs4237808, rs499646, rs11639774) in predicting TD for individuals taking typical antipsychotics. However, for individuals taking atypical antipsychotics, there was a significant genetic contribution to predicting TD cases beyond clinical factors (See Fig. $4 \mathrm{~b}, \mathrm{c}, \Delta \chi^{2}=38.96$, $\mathrm{df}=4$, $\left.p=7.09 \times 10^{-8}\right)$. It is notable that after adjusting for population stratification, database, and sex, Age of Onset $(\mathrm{OR}=$ $1.071, \quad 95 \% \quad \mathrm{CI}=1.029-1.114, \quad$ p.adj $\left.=1.96 \times 10^{-2}\right)$ and Duration of illness $(\mathrm{OR}=1.105,95 \% \mathrm{CI}=1.069-1.142$, 
p.adj $=1.18 \times 10^{-7}$ ) significantly predicted TD. Beyond clinical factors, two of the top SNPs rs499646 [TNFRSF1B] $\left(\mathrm{OR}=2.957,95 \% \mathrm{CI}=1.669-5.239, \quad\right.$ p.adj $\left.=5.86 \times 10^{-3}\right)$ and $\mathrm{rs} 4237808$ [CALCOCO1] $(\mathrm{OR}=2.937,95 \% \mathrm{CI}=$ 1.713-5.038, p.adj $=2.62 \times 10^{-3}$ ) identified in the earlier GWAS significantly predicted the emergence of TD. Note that p.adj are Bonferroni adjusted $p$-values for 29 variables in the logistic prediction model (See Supplementary Information for further details).

\section{Lookup of past tardive dyskinesia studies}

Variants previously found to be associated with TD were meta-analyzed with results from the current GWAS

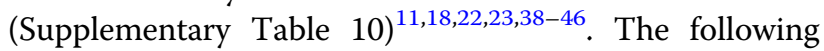
variants, independent from CATIE were replicated in the current study CYP1A2 (rs762551, $N=1725, P=0.018$ ), DRD1 (rs4532, $N=1788, P=0.045)$, GRIN2A (rs1345423, $N=1837, P=0.012), G R I N 2 B(\mathrm{rs} 2192970, N=1057, P=$ $0.0018)$, HSPG2 (rs878949 (proxy), $N=1572, P=0.0051$ ), and DPP6 (rs6977820, $N=1701, P=0.00068$ ). These genes implicated pathways involving drug metabolism, dopamine, and glutamate.

\section{Discussion}

To our knowledge, here we report the largest GWAS for TD. A single novel locus at 16q24.1 was found to be associated with TD. We also identified three loci (1p36.22, 6q23.2, and 12q13.13) with suggestive evidence of association to TD. Of these, putative causal variants were identified for three of the loci. The top GWAS hit at $16 \mathrm{q} 24.1$, in the GSE1 coiled-coil protein gene, encodes for a proline rich protein which was reported to be a subunit of BRAF35-HDAC (BHC) histone deacetylase complex ${ }^{51}$. This gene has been known to be implicated in the proliferation, migration, and invasion of breast cancer cells ${ }^{52}$. A lookup in the GWAS catalog revealed GSE1 was also associated at GWAS significance with platelet count and distribution ${ }^{53}$, and suggestive GWAS significance with amyotrophic lateral sclerosis $^{54}$ and sulfasalazine-induced agranulocytosis ${ }^{55}$. GSE1 contributes to a geneset that are predicted targets of a microRNA biomarker for schizophrenia ${ }^{56}$.

Other suggestive associations with TD included TNFRSF1B (1p36.22), EPB41L2 (6q23.2), and CALCOCO1 (12q13.13). The tumor necrosis factor receptor superfamily member $1 \mathrm{~B}$ (TNFRSF1B) is a protein-coding gene that mediates antiapoptotic signaling. Expression of TNFRSF1B is specific to cells in the immune systems, specific neuronal subtypes, certain $\mathrm{T}$-cells subtypes, and endothelial cells ${ }^{57}$. This appears to be supported by results from the competitive geneset enrichment analysis, albeit nonsignificant, revealed top pathways that implicated immunoglobulin production, chemokine receptors, and monocytes. These findings appear to support existing theories on the role of immune and inflammation in the pathogenesis of $\mathrm{TD}^{11,12}$.

The erythrocyte membrane protein band 4.1 like 2 (EPB41L2) is involved in actin and cytoskeletal protein binding. More recently, EPB41L2 deficiency was found to result in myelination abnormalities in the peripheral nervous system, leading to motor neuropathy in a mice study $^{58}$. This putative association of EPB41L2 deficiency is consistent with the direction of effect found in our GWAS results, suggesting that the expression of EPB41L2 might confer a protective effect against motor neuropathy. Functional enrichment in and around the EPB41L2 based on the MetaXcan finding is intriguing, further research is needed to dissect the function of EPB41L2 in TD pathophysiology.

The calcium binding and coiled-coil domain 1 (CALCOCO1) is a protein-coding gene that is involved in the activation of transcriptional activities of targets genes in the Wnt signaling pathway, neuronal receptor and aryl hydrocarbon receptor $(\mathrm{AhR})^{59}$. Notably, one function of the $\mathrm{AhR}$, a ligand-based transcription factor, is the regulation of transcriptional activity for drug metabolizing enzymes, including the family of cytochrome P450 (CYP) genes $^{60}$. The family of CYP has been postulated to be a candidate for TD susceptibility ${ }^{11}$. Specifically, CYP enzymes, such as CYP1A2, CYP2D6, and CYP3A4, metabolize antipsychotics (e.g., clozapine, olanzapine, and haloperidol) and antidepressants (e.g., fluvoxamine) ${ }^{60}$. Meta-analysis of CYP1A2 from past and current study revealed that this gene was significantly associated with TD at trend level $(P<0.05$; Supplementary Table 10).

Follow-up post-hoc investigation showed that individuals that were on Typical antipsychotics were expectedly more likely to be a TD case. We also demonstrated that clinical factors such as Age of Onset and Duration of illness of schizophrenia also significantly predicted TD. However, beyond clinical factors, top SNPs within $C A L$ COCO1 and TNFRSF1B were responsible for accounting for nearly three times the risk of having TD and that joint clinical and genetic model accounted for greater than $75 \%$ of variance for individuals having TD and were on atypical antipsychotics. Nevertheless, further investigation is required to confirm the replicability and generalizability of the observed phenomenon.

Polygenic risk score analysis on traits with polygenic architecture revealed overlapping genetic polygenic risk of TD and ALS. The shared protein misfolding pathway between TD and ALS is consistent with findings from our MAGMA pathway analysis. Post-hoc lookups of known ALS genes that might be associated with TD revealed that an F-Box protein-encoding gene $F B X O 15$, chemo/neurokine encoding gene $F A M 19 A 1$, and neuropeptide encoding gene, NPS. Proteinopathies commonly implicated in neurodegenerative conditions have been proposed to also 
underlie $\mathrm{TD}^{61,62}$. Prior reports have implicated the accumulative role of proteinopathies in neurotoxicity, synaptic dysfunction, and its bidirectional effect with oxidative stress, neuroinflammation, and its consequence on the immune system ${ }^{61,62}$. We do note, however, that genetic overlap between TD and other seemingly related traits is generally weak, such as neurodegenerative conditions and autoimmune conditions. Nevertheless, Schizophrenia and Rheumatoid Arthritis were close to the multiple correction threshold. Future work in more powered samples may be carried out to investigate how the potential biological mechanisms for ALS, rheumatoid arthritis, and schizophrenia might be related to TD. We note that potential ancestry differences in LD patterns and allele frequencies of the target and training dataset could result in poorer polygenic prediction. The generally small pharmacogenomic datasets utilized in the context of this report, and the use of multiple reference panels (i.e., HapMap and 1000 genomes project) could have contributed to the variability of the results. Nevertheless, pharmacogenomic datasets such as those reported here continue to allow more clues to be shed regarding the potential biological mechanisms that underlie complex clinical effects that arise from the prescription of psychotropic medication.

Emergent results from the current report demonstrate the polygenic architecture of TD. While the current study remains underpowered for GWAS analysis (Supplementary Fig. 12), we present the largest GWAS of TD to date. The findings reported here suggest that multiple overlapping biological systems might contribute to the etiopathogenesis of the condition. Taken together, results suggest that TD is associated with significant proteinopathies, disrupted neuronal function within the brain and potentially including muscular innervation, existing in a background of inflammation. Further work is necessary to identify the cascade of biological disruptions and events that trigger TD symptomatology. Further work is needed to replicate findings in the current report and further unravel the biology of these risk variants and pathways identified.

\section{Web resources}

FUMA, http://fuma.ctglab.nl/.

GAS Power Calculator, http://csg.sph.umich.edu/ abecasis/cats/gas_power_calculator/index.html

GCTA, https://cnsgenomics.com/software/gcta/

GEMMA, http://www.xzlab.org/software.html

GWAS Catalog, https://www.ebi.ac.uk/gwas/

LD-HUB, http://ldsc.broadinstitute.org/

MAGMA, https://ctg.cncr.nl/software/magma

METAL, https://genome.sph.umich.edu/wiki/METAL_

Documentation

MetaXcan, https://github.com/hakyimlab/MetaXcan
Michigan Imputation Server, https://imputationserver. sph.umich.edu/index.html

MSigDB, http://software.broadinstitute.org/gsea/msigdb PAINTOR, https://github.com/gkichaev/PAINTOR_V3.0 PLINK1.9, https://www.cog-genomics.org/plink2

PRSice2, https://github.com/choishingwan/PRSice/wiki PredictDB, http://predictdb.org/

R, https://www.r-project.org/

SNAP, http://www.broad.mit.edu/mpg/snap/

Variant Effect Predictor, https://www.ensembl.org/vep

\section{Acknowledgements}

This research is supported by grants from the Ministry of Health Singapore, National Medical Research Council (Grant No.: NMRC/TCR/003/2008, NMRC/CG/ 004/2013). M.L. is supported by the National Medical Research Council Research Training Fellowship (Grant No:: MH095:003/008-1014). The work was (partly) supported by an A*STAR SPF grant to the SAPhIRE program. J. J.L. acknowledges the BMRC Central Research Fund (UIBR), Agency for Science, Technology and Research, Singapore and the BMRC Strategic Position Fund (SPF2014/001),

Agency for Science, Technology and Research, Singapore. We thank the NIH for providing limited access datasets for the NIMH CATIE (ClinicalTrials.gov identifier NCT00014001, NIMH contract \#N01MH90001). We would like to express our gratitude to Professor Arinami Tadao for his valuable comments. We also thank all participants and researchers who contributed to the collection of this data.

\section{Author details}

${ }^{1}$ Research Division, Institute of Mental Health, Singapore, Singapore. ${ }^{2}$ Zucker Hillside Hospital, New York, NY, USA. ${ }^{3}$ Feinstein Institutes of Medical Research, New York, NY, USA. ${ }^{4}$ Tanenbaum Centre for Pharmacogenetics, Center for Addiction and Mental Health; Department of Psychiatry, Institute of Medical Science, Laboratory Medicine and Pathobiology, University of Toronto, Toronto, ON, Canada. ${ }^{5}$ Genome Institute of Singapore, Singapore, Singapore. ${ }^{6}$ Centre of Excellence in Mental Health, ABVIMS \& Dr Ram Manohar Lohia Hospital, New Delhi, India. ${ }^{7}$ Department of Genetics, University of Delhi south campus, New Delhi, India. ${ }^{8}$ Department of Psychiatry, Graduate School of Medicine, Nagoya University, Nagoya, Aichi, Japan. ${ }^{9}$ Department of Psychiatry and Psychobiology, Graduate School of Medicine, Nagoya University, Nagoya, Aichi, Japan. ${ }^{10}$ West Region, Institute of Mental Health, Singapore, Singapore. ${ }^{11}$ Department of Psychosis, Institute of Mental Health, Singapore, Singapore. ${ }^{12}$ Donald and Barbara Zucker School of Medicine at Hofstra/Northwell, New York, NY, USA. ${ }^{13}$ Yong Loo Lin School of Medicine, National University of Singapore, Singapore, Singapore. ${ }^{14}$ Neuroscience and Mental Health, Lee Kong Chian School of Medicine, Nanyang Technological University, Singapore, Singapore

\section{Author contributions}

M.L. and K.L. ran the analysis and drafted the manuscript, J.J.L. and J.L. designed the study and supervised preparation of the manuscript. All other authors were involved in the drafting and approved the final manuscript. J.L. takes responsibility for data access.

\section{Conflict of interest}

The authors declare no competing interests.

\section{Publisher's note}

Springer Nature remains neutral with regard to jurisdictional claims in published maps and institutional affiliations.

Supplementary information The online version contains supplementary material available at https://doi.org/10.1038/s41398-021-01471-y.

Received: 17 November 2020 Revised: 20 April 2021 Accepted: 13 May 2021

Published online: 08 June 2021 


\section{References}

1. Correll, C. U., Kane, J. M. \& Citrome, L. L. Epidemiology, prevention, and assessment of tardive dyskinesia and advances in treatment. J. Clin. Psychiatry 78, 1136-1147 (2017).

2. Zai, C. C. et al. Genetics of tardive dyskinesia: promising leads and ways forward. J. Neurol. Sci. https://doi.org/10.1016/j.jns.2018.02.011 (2018).

3. Weiner, W. J. Drug-induced Movement Disorders. in Encyclopedia of Movement Disorders (ed. Metman, K. K. A. L.) 340-347 (Elsevier, 2010).

4. Correll, C. U. \& Schenk, E. M. Tardive dyskinesia and new antipsychotics. Curr. Opin. Psychiatry 21, 151-156 (2008).

5. Lee, J., Jiang, J., Sim, K. \& Chong, S.-A. The prevalence of tardive dyskinesia in Chinese Singaporean patients with schizophrenia: revisited. J. Clin. Psychopharmacol. 30, 333-335 (2010).

6. Zhang, X. Y. et al. Gender differences in the prevalence, risk and clinical correlates of tardive dyskinesia in Chinese schizophrenia. Psychopharmacology 205, 647-654 (2009)

7. Chong, S.-A., Tay, J. A. M., Subramaniam, M., Pek, E. \& Machin, D. Mortality rates among patients with schizophrenia and tardive dyskinesia. J. Clin. Psychopharmacol. 29, 5-8 (2009).

8. Wu, J. Q. et al. Cognition impairment in schizophrenia patients with tardive dyskinesia: association with plasma superoxide dismutase activity. Schizophr. Res. 152, 210-216 (2014)

9. Adrianzén, C. et al. Relative association of treatment-emergent adverse events with quality of life of patients with schizophrenia: post hoc analysis from a 3-year observational study. Hum. Psychopharmacol. 25, 439-447 (2010).

10. Tenback, D. E., van Harten, P. N., Slooff, C. J. \& van Os, J. Evidence that early extrapyramidal symptoms predict later tardive dyskinesia: a prospective analysis of 10,000 patients in the European Schizophrenia Outpatient Health Outcomes (SOHO) study. Am. J. Psychiatry 163, 1438-1440 (2006).

11. Lanning, R. K., Zai, C. C. \& Müller, D. J. Pharmacogenetics of tardive dyskinesia: an updated review of the literature. Pharmacogenomics 17, 1339-1351 (2016).

12. Lee, H.-J. \& Kang, S.-G. Genetics of tardive dyskinesia. Int. Rev. Neurobiol. 98 231-264 (2011).

13. Sarró, S. et al. Structural brain changes associated with tardive dyskinesia in schizophrenia. Br. J. Psychiatry 203, 51-57 (2013).

14. Bakker, P. R., van Harten, P. N. \& van Os, J. Antipsychotic-induced tardive dyskinesia and polymorphic variations in COMT, DRD2, CYP1A2 and MnSOD genes: a meta-analysis of pharmacogenetic interactions. Mol. Psychiatry 13, 544-556 (2008)

15. Tsai, H.-T. et al. A candidate gene study of tardive dyskinesia in the CATIE schizophrenia trial. Am. J. Med. Genet. B Neuropsychiatr. Genet. 153B, 336-340 (2010).

16. Thelma, B. K., Srivastava, V. \& Tiwari, A. K. Genetic underpinnings of tardive dyskinesia: passing the baton to pharmacogenetics. Pharmacogenomics $\mathbf{9}$ 1285-1306 (2008).

17. Bakker, P. R., van Harten, P. N. \& van Os, J. Antipsychotic-induced tardive dyskinesia and the Ser9Gly polymorphism in the DRD3 gene: a meta analysis. Schizophr. Res. 83, 185-192 (2006).

18. Roberto Bakker, P. et al. Antipsychotic-induced movement disorders in longstay psychiatric patients and 45 Tag SNPs in 7 candidate genes: a prospective study. PLOS ONE 7, e50970 (2012).

19. Greenbaum, L., Alkelai, A., Rigbi, A., Kohn, Y. \& Lerer, B. Evidence for association of the GLI2 gene with tardive dyskinesia in patients with chronic schizophrenia. Mov. Disord. 25, 2809-2817 (2010).

20. Inada, T. et al. Pathway-based association analysis of genome-wide screening data suggest that genes associated with the gamma-aminobutyric acid receptor signaling pathway are involved in neuroleptic-induced, treatmentresistant tardive dyskinesia. Pharmacogenet. Genomics 18, 317-323 (2008).

21. Syu, A. et al. Association of the HSPG2 gene with neuroleptic-induced tardive dyskinesia. Neuropsychopharmacology 35, 1155-1164 (2010)

22. Tanaka, S. et al. DPP6 as a candidate gene for neuroleptic-induced tardive dyskinesia. Pharmacogenomics J. 13, 27-34 (2013).

23. Greenbaum, L., Alkelai, A., Zozulinsky, P., Kohn, Y. \& Lerer, B. Support for association of HSPG2 with tardive dyskinesia in Caucasian populations. Pharmacogenomics J. 12, 513 (2011).

24. Lieberman, J. A. et al. Effectiveness of antipsychotic drugs in patients with chronic schizophrenia. N. Engl. J. Med. 353, 1209-1223 (2005)

25. Stroup, T. S. et al. The National Institute of Mental Health Clinical Antipsychotic Trials of Intervention Effectiveness (CATIE) project: schizophrenia trial design and protocol development. Schizophr. Bull. 29, 15-31 (2003).
26. Sullivan, P. F. et al. Genomewide association for schizophrenia in the CATIE study: results of stage 1. Mol. Psychiatry 13, 570-584 (2008).

27. Guy. W. ECDEU Assessment Manual for Psychopharmacology. 534-537 (U.S Dept. of Health, Education, and Welfare, Public Health Service, Alcohol, Drug Abuse, and Mental Health Administration, National Institute of Mental Health, Psychopharmacology Research Branch, Division of Extramural Research Programs, 1976).

28. Schooler, N. R. \& Kane, J. M. Research diagnoses for tardive dyskinesia. Arch. Gen. Psychiatry 39, 486-487 (1982).

29. Zhou, X. \& Stephens, M. Genome-wide efficient mixed-model analysis for association studies. Nat. Genet. 44, 821-824 (2012).

30. Willer, C. J., Li, Y. \& Abecasis, G. R. METAL: fast and efficient meta-analysis of genomewide association scans. Bioinformatics 26, 2190-2191 (2010).

31. Watanabe, K., Taskesen, E., van Bochoven, A. \& Posthuma, D. Functional mapping and annotation of genetic associations with FUMA. Nat. Commun. 8, 1826 (2017)

32. de Leeuw, C. A., Mooij, J. M., Heskes, T. \& Posthuma, D. MAGMA: Generalized gene-set analysis of GWAS data. PLoS Comput. Biol. 11, e1004219 (2015).

33. Barbeira, A. et al. MetaXcan: Summary statistics based gene-level association method infers accurate PrediXcan results. bioRxiv https://doi.org/10.1101/ 045260 (2016).

34. Kichaev, G. et al. Integrating functional data to prioritize causal variants in statistical fine-mapping studies. PLoS Genet. 10, e1004722 (2014).

35. Kichaev, G. \& Pasaniuc, B. Leveraging functional-annotation data in transethnic fine-mapping studies. Am. J. Hum. Genet. 97, 260-271 (2015).

36. Yang, J., Lee, S. H., Goddard, M. E. \& Visscher, P. M. GCTA: a tool for genomewide complex trait analysis. Am. J. Hum. Genet. 88, 76-82 (2011).

37. Euesden, J., Lewis, C. M. \& O'Reilly, P. F. PRSice: Polygenic Risk Score software Bioinformatics 31, 1466-1468 (2015).

38. Fedorenko, O. Y. et al. Association study indicates a protective role of phosphatidylinositol-4-phosphate-5-kinase against tardive dyskinesia. Int. J. Neuropsychopharmacol. 18, 1-6 (2014).

39. Hsieh, C.-J., Chen, Y.-C., Lai, M.-S., Hong, C.-J. \& Chien, K.-L. Genetic variability in serotonin receptor and transporter genes may influence risk for tardive dyskinesia in chronic schizophrenia. Psychiatry Res. 188, 175-176 (2011).

40. Ivanova, S. A. et al. Cytochrome P450 1A2 co-determines neuroleptic load and may diminish tardive dyskinesia by increased inducibility. World J. Biol. Psychiatry 16, 200-205 (2015).

41. Ivanova, S. A. et al. Dehydroepiandrosterone sulphate as a putative protective factor against tardive dyskinesia. Prog. Neuropsychopharmacol. Biol. Psychiatn 50, 172-177 (2014).

42. Ivanova, S. A. et al. NMDA receptor genotypes associated with the vulnerability to develop dyskinesia. Transl. Psychiatry 2, e67 (2012).

43. Lai, I-C. et al. Analysis of genetic variations in the dopamine D1 receptor (DRD1) gene and antipsychotics-induced tardive dyskinesia in schizophrenia. Eur. J. Clin. Pharmacol. 67, 383-388 (2011).

44. Son, W.-Y. et al. Gaba transporter SLC6A11 gene polymorphism associated with tardive dyskinesia. Nord. J. Psychiatry 68, 123-128 (2014).

45. Tiwari, A. K. et al. Association study of cannabinoid receptor 1 (CNR1) gene in tardive dyskinesia. Pharmacogenomics J. 12, 260-266 (2012).

46. Zai, C. C. et al. Association study of the vesicular monoamine transporter gene SLC18A2 with tardive dyskinesia. J. Psychiatr. Res. 47, 1760-1765 (2013).

47. Johnson, A. D. et al. SNAP: a web-based tool for identification and annotation of proxy SNPs using HapMap. Bioinformatics 24, 2938-2939 (2008).

48. Subramanian, A. et al. Gene set enrichment analysis: a knowledge-based approach for interpreting genome-wide expression profiles. Proc. Natl Acad. Sci. USA 102, 15545-15550 (2005).

49. GTEx Consortium. Human genomics. The Genotype-Tissue Expression (GTEx) pilot analysis: multitissue gene regulation in humans. Science 348, 648-660 (2015).

50. McLaren, W. et al. The ensembl variant effect predictor. Genome Biol. 17, 122 (2016).

51. Hakimi, M.-A., Dong, Y., Lane, W. S., Speicher, D. W. \& Shiekhattar, R. A candidate $\mathrm{X}$-linked mental retardation gene is a component of a new family of histone deacetylase-containing complexes. J. Biol. Chem. 278, 7234-7239 (2003).

52. Chai, P. et al. GSE1 negative regulation by miR-489-5p promotes breast cancer cell proliferation and invasion. Biochem. Biophys. Res. Commun. 471, 123-128 (2016). 
53. Astle, W. J. et al. The allelic landscape of human blood cell trait variation and links to common complex disease. Cell 167, 1415-1429.e19 (2016).

54. Xie, T. et al. Genome-wide association study combining pathway analysis for typical sporadic amyotrophic lateral sclerosis in Chinese Han populations. Neurobiol. Aging 35, 1778.e9-1778.e23 (2014).

55. Wadelius, M. et al. Sulfasalazine-induced agranulocytosis is associated with the human leukocyte antigen locus. Clin. Pharmacol. Ther. https://doi.org/10.1002/ cpt.805 (2017).

56. Hass, J. et al. Associations between DNA methylation and schizophreniarelated intermediate phenotypes - a gene set enrichment analysis. Prog. Neuropsychopharmacol. Biol. Psychiatry 59, 31-39 (2015).

57. Naudé, P. J. W., den Boer, J. A., Luiten, P. G. M. \& Eisel, U. L. M. Tumor necrosis factor receptor cross-talk. FEBS J. 278, 888-898 (2011).
58. Saitoh, Y., Ohno, N., Yamauchi, J., Sakamoto, T. \& Terada, N. Deficiency of a membrane skeletal protein, $4.1 \mathrm{G}$, results in myelin abnormalities in the peripheral nervous system. Histochem. Cell Biol. 148, 597-606 (2017).

59. Yang, C. K. Kim, J. H. \& Stallcup, M. R. Role of the N-terminal activation domain of the coiled-coil coactivator in mediating transcriptional activation by betacatenin. Mol. Endocrinol. 20, 3251-3262 (2006).

60. Ramadoss, P., Marcus, C. \& Perdew, G. H. Role of the aryl hydrocarbon receptor in drug metabolism. Expert Opin. Drug Metab. Toxicol. 1, 9-21 (2005).

61. Ciechanover, A. \& Kwon, Y. T. Degradation of misfolded proteins in neurodegenerative diseases: therapeutic targets and strategies. Exp. Mol. Med. 47, e147 (2015)

62. Sweeney, P. et al. Protein misfolding in neurodegenerative diseases: implications and strategies. Transl. Neurodegener. 6, 6 (2017) 